Research Article

\title{
Artificial Neural Network Modeling of the Effect of Cutting Conditions on Cutting Force Components during Orthogonal Turning
}

\author{
Sanjib Banerjee $\dot{\mathrm{A}}^{*}$, Angshuman Deka $^{\dot{\mathrm{A}}}$, Gitartha Dev Sarmah ${ }^{\dot{\mathrm{A}}}$, Nitish Bhardwaj $^{\dot{\mathrm{A}}}$ \\ ${ }^{\dot{A}}$ Department of Mechanical Engineering, Tezpur University, 784028, Tezpur, India
}

Accepted 16 January 2014, Available online 01 February 2014, Special Issue-2, (February 2014)

\begin{abstract}
Variation in cutting force components $\left(F_{X}, F_{Y}\right.$ and $F_{Z}$ in three mutually perpendicular directions $X, Y$ and $\left.Z\right)$ with cutting conditions viz. speed (v), feed $(f)$ and depth of cut (d) during orthogonal turning of mild steel specimen using a HSS cutting tool was investigated in an automatic lathe machine. During the turning process, the cutting forces experienced insignificant variations with change in speed. Cutting force values were however observed to considerably increase with increase in feed and depth of cut. Subsequently these cutting forces could be modelled as function of the cutting conditions viz. $v, f$ and $d$, by artificial neural network (ANN). The cutting force values modelled and subsequently predicted at various cutting conditions within the specified domain have been successfully correlated with the experimental results and literature review, with fairly good accuracy. During the validation process, it was possible to predict $100 \%, 94 \%$ and $100 \%$ of the cutting force values for $F_{X}, F_{Y}$ and $F_{Z}$, respectively within a percentage deviation of $\pm 10 \%$. This observation highlights the superior prediction capability of ANN technique in the current research area.
\end{abstract}

Keywords: Cutting Forces; Cutting Conditions; Artificial neural network; Orthogonal Turning; Mild Steel.

\section{Introduction}

It has been realized by most of the manufacturing engineers and researchers that in order to optimize the efficiency of metal cutting operations, efficient quantitative and predictive models that establish a relationship between a big group of independent input parameters and output variables are required for the wide spectrum of manufacturing processes, cutting tools and conditions, and engineering materials currently used in the industries (Wong, et al, 1999). Furthermore, it has been observed that proper optimization of the output variables, such as tool life, cutting forces, surface roughness, etc., through the optimization of input parameters like cutting speed, feed rate and depth of cut, results in a significant improvement in the economic performance of machining operations ( $\mathrm{Li}$ and $\mathrm{Li}, 2002$ ). One of these output variables that may either directly or indirectly affect the performance of other variables such as tool wear rate, machined surface characteristics and machining costs is cutting force (Kadirgama, et al, 2008). Cutting forces directly relate with the tool wear as higher the forces experienced by the tool; higher will be the tool wear rate. Subsequently the cutting forces also have an impact on the tool life, cutting cost, machine vibrations, and dimensional accuracies, cutting power, and machined surface characteristics. The same fact reveals the significance of investigating and modeling of cutting forces (Yun and Cho, 2001). During orthogonal turning of a cylindrical

*Corresponding author: Sanjib Banerjee

DOI: http://dx.doi.org/10.14741/ijcet/spl.2.2014.23 work piece, the cutting force experienced by the tool may be considered as functions of the cutting conditions viz. $v$, $f$ and $d$. These force values of a particular material can be modeled from the experimental results, by using artificial neural network (ANN) (Suksawat, 2010). The cutting force components can then be successfully predicted for any combination of the input variables within the specific domain. Such prediction provides immense industrial help in formulating the cutting conditions on the basis of cutting forces developed and thus deciding the subsequent manufacturing parameters required during orthogonal turning of mild steel. Out of these three forces, $F_{\mathrm{Y}}$ shows the most considerable effect during orthogonal turning operation as power required for machining is determined by the cutting force component, $F_{\mathrm{Y}}$.

A neural network is similar to the biological nervous system, which is basically a connectionist system, in which various nodes called neurons are interconnected. An artificial neural network (ANN) can be defined as a model of reasoning similar to the human brain, where a large amount of complex information can be stored and processed simultaneously by each neuron along the entire domain. A typical neuron receives one or more input signals and provides an output signal depending on the processing function of the neuron. This output is transferred to connected neurons in varying intensities, the signal intensity being decided by the weights assigned. ANN has the advantages of (i) modelling the data where the input-output relation is either unknown or nonlinear, (ii) adaptive learning during training (iii) real time 
applications aided by a very fast computational speed. The conventional computational techniques follow an algorithmic approach, where a set of instructions in the specified order is followed to solve a problem. The relationship between each stage is required for solving the problem. On the other hand, neural network technique is data driven and the solution can be obtained even if the exact relationship is unknown. This can be obtained if a number of input-output data sets are available. Once the ANN architecture is fixed, the output for any combination of input variables can be predicted.

The most popular neural networks are feed forward networks. Feed forward network architecture consists of three distinct layers: the input layer, the hidden layer(s), and the output layer. Each layer consists of a number of neurons. The output from the neurons of one layer is transferred as input to neurons of the succeeding layer. The first layer, called an input layer, receives data from the outside world. The second layer, called the hidden layer, does not have any direct contact with the outside world and is used to help in extracting higher-level features and to facilitate generalization of outputs. The last layer is the output layer, which sends information out to users. For a given input vector, it generates the output vector by a forward pass. The data are fed to the network at the input layer and propagated with weights and activation functions to the output layer to provide the response. Once the data at the output neuron is reached, the mean squared error (MSE), which is the difference between the network output vector and the known target vector, is computed and back-propagated to modify the weights for the entire network.

For orthogonal turning of mild steel, the input layer of ANN consists of three neurons representing the three cutting conditions viz., $v, f$ and $d$, as shown in Fig. 1. The output layer on the other hand consists of one neuron representing $F_{\mathrm{X}}, F_{\mathrm{Y}}$ or $F_{\mathrm{Z}}$. Once the proper network architecture is determined, $F_{\mathrm{X}}, F_{\mathrm{Y}}$ and $F_{\mathrm{Z}}$ can be successfully predicted for any combination of the input variables within the specified domain (Banerjee, et al, 2011).

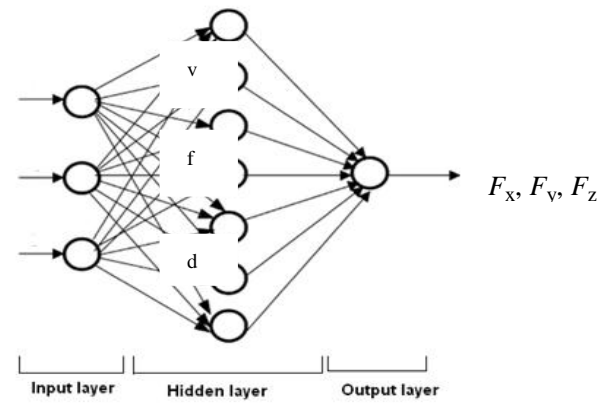

Fig. 1 A typical neural network architectre for modeling cutting force

\section{Experimental procedures}

Four numbers of mild steel rods of $24 \mathrm{~mm}$ diameter were taken up for orthogonal turning operation at four different depths of cuts. The total of 64 readings (the combination of four speeds, four feeds and four depths of cuts) could not be taken from a single rod because this would have required a rod of substantial length and resulted in considerable vibration. So, it was decided to take 16 readings in a rod of particular depth of cut. The details regarding the cutting conditions investigated have been shown in Table 1 . The turning operation was carried out in an automatic lathe machine (MAKE: HMT MACHINE TOOLS LIMITED, MODEL: NH26). High speed steel (HSS) was used as the cutting tool material. HSS are basically high carbon steels with significant quantities of $\mathrm{W}, \mathrm{Mo}, \mathrm{Cr}, \mathrm{V}$ and Co.

During each turning operation, the cutting forces viz. $F_{\mathrm{X}}, F_{\mathrm{Y}}$ and $F_{\mathrm{Z}}$ were measured in three mutually perpendicular directions, $X, Y$ and $Z$, as indicated:

$F_{\mathrm{X}}=$ Acts in the direction of the feed (axial direction) and is called the feed force. If unchecked it can lead to dimensional inaccuracy and vibration.

$F_{\mathrm{Y}}=$ Acts in the tangential direction and is called the cutting force or power component.

$F_{\mathrm{Z}}=$ It acts in the radial direction and is called the thrust force (Jain, 2007; Lal 2007).

Out of these three force components experienced during an orthogonal turning operation, the maximum value is for cutting force followed by thrust force and feed force. Thus cutting force has the maximum effect on the tool wear and tool life (Altintas, 2000; Gokkaya 2010). Hence for optimization purpose, this force, $F_{\mathrm{Y}}$, is most significant to be analyzed.

The cutting forces were measured by a piezoelectric tool dynamometer (MAKE: IEICOS LATHE TOOL DYNAMOMETER, MODEL: 620B) along with a digital multi component force indicator (MODEL: 652). In order to eliminate experimental errors, five consecutive readings of each parameter were taken at a particular set of cutting condition and the average of these five was considered for subsequent modeling.

After evaluating cutting force values for different cutting conditions, the same were modeled by artificial neural network (ANN). The ANN modeling was carried out by the multiple layer perception (MLP) feed forward back propagation network. The cutting conditions viz. $v, f$ and $d$ representing three neurons in the input layer and cutting force components representing the neuron in the output layer formed the data sets for training the network. 'Neural Network' tool box available with the MATLAB (Release 7) software package was used for the present modeling. Training of the neural network was done using the ANN tool kit of MATLAB software, using 'TRAINLM' function. 'TRAINLM' is a network training function that updates weights and bias values in a back propagation algorithm according to Levenberg-Marquard optimization. Levenberg-Marquardt algorithm is a highly efficient method for solving non-linear optimization problems (Robi and Dixit, 2003). Single layer hidden neurons were used in the network architecture. The number of neurons in the hidden layer, the transfer functions at the input-to-hidden layer and hidden-to-output layer were optimized by trial and error method during the network training and testing stages. The mean square error (MSE) during the training and testing was determined for each trial. The network architecture was finally frozen 
based on the minimum MSE value obtained during both the training and testing stages. Out of the total 64 number of data sets, 32 and 20 data sets were used for respectively training and testing operations. 12 data sets from the remaining were used for validating the trained network architecture. Once the proper network architecture was arrived at, the cutting force components could be successfully predicted for any combination of input variables $(v, f$ and $d$ ), within the specified domain.

Table 1 Different Cutting Conditions for prediction of Cutting Forces

\begin{tabular}{lll}
\hline Depth of Cut $(\mathrm{mm})$ & Feed(mm/rev) & Speed(RPM) \\
\hline $0.24,0.50,0.64$ and & $0.05,0.20,0.52$ and & $114,325,550$ and \\
0.80 & 0.80 & 930
\end{tabular}

\section{Results and Discussion}

\subsection{Experimental and Predicted Curves for Cutting Forces}

The experimental values were obtained from the turning operation of mild steel. The results revealed that the cutting force almost remains constant with change in cutting speed while it increases with increase in feed and depth of cut, as shown in Figure 2. These trends are well correlated with the available open literature and earlier studies (Satyanarayana, 2011; Malagi and Rajesh, 2012; Kanthraj, et al, 2012).

\subsection{Modeling and Prediction of Cutting forces by Artificial Neural Network}

In order to obtain the prediction of cutting forces with variation in cutting parameters, artificial neural network (ANN) modeling was carried out. The cutting force components being functions of the cutting conditions can be expressed as:

$F_{\mathrm{X}}, F_{\mathrm{Y}}, F_{\mathrm{Z}}=F(v, f, d)$

The ANN architecture used in present modeling consists of three layers, viz. an input layer of three neurons representing the cutting conditions of $v, f$ and $d$, a hidden layer of $n$ numbers of neurons and an output layer with one neuron representing the cutting force components. Appropriate values for the weight and bias of the network architecture were arrived at by training the network using several data sets. Out of total number of 64 input-output data sets obtained from experimentations, it was decided to use 32, 20 and 12 data sets for the network training, testing and validation purposes respectively. The validation data sets were not used earlier for the testing or training purposes. Both training and testing of the network were carried our independently. A number of numerical trials were carried out with single hidden layer neural network. The tansig, logsig and purelin transfer functions were tried with while simultaneously varying the number of neurons in the hidden layer, in order to arrive at the best network architecture. The sum squared training error goal for $F_{\mathrm{X}}, F_{\mathrm{Y}}$ and $F_{\mathrm{Z}}$ was fixed at 0.00001 . After a number of trials with various initial weights and biases, the best
Table 2 Best fit network architectures for the investigated cutting force components

\begin{tabular}{llll}
\hline $\begin{array}{l}\text { Force } \\
\text { Component }\end{array}$ & Hidden & $1^{\text {st }}$ Transfer & $2^{\text {nd }}$ Transfer \\
& Neurons & Function & Function \\
\hline$F_{\mathrm{X}}$ & 5 & logsig & purelin \\
\hline$F_{\mathrm{Y}}$ & 6 & logsig & purelin \\
\hline$F_{\mathrm{Z}}$ & 4 & logsig & purelin \\
\hline
\end{tabular}

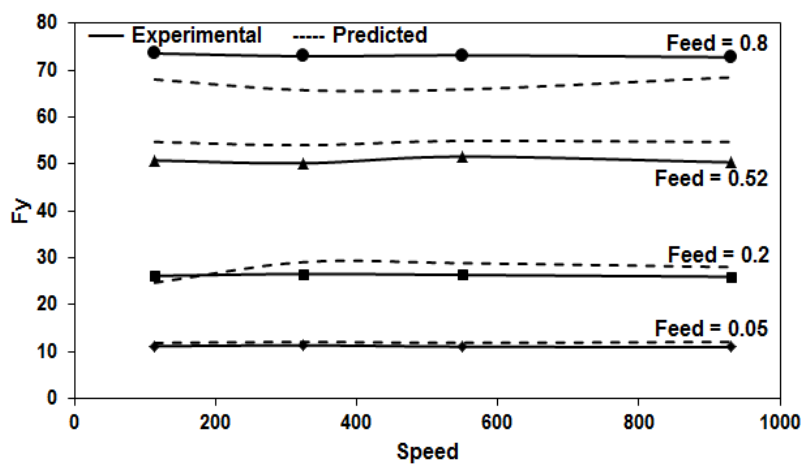

(a)

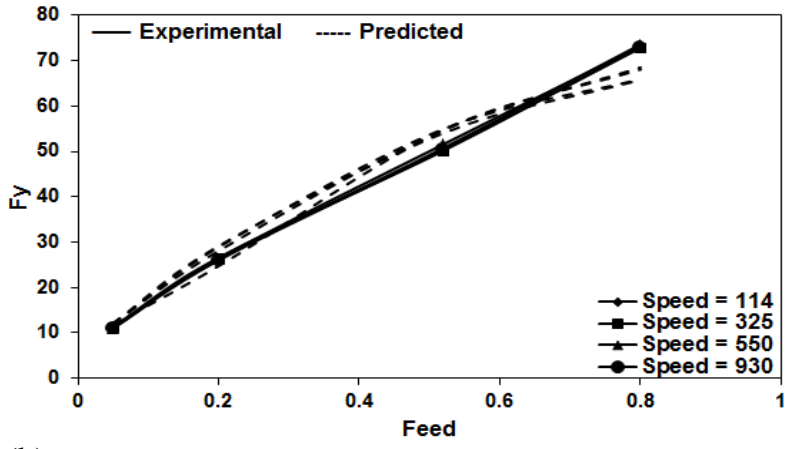

(b)

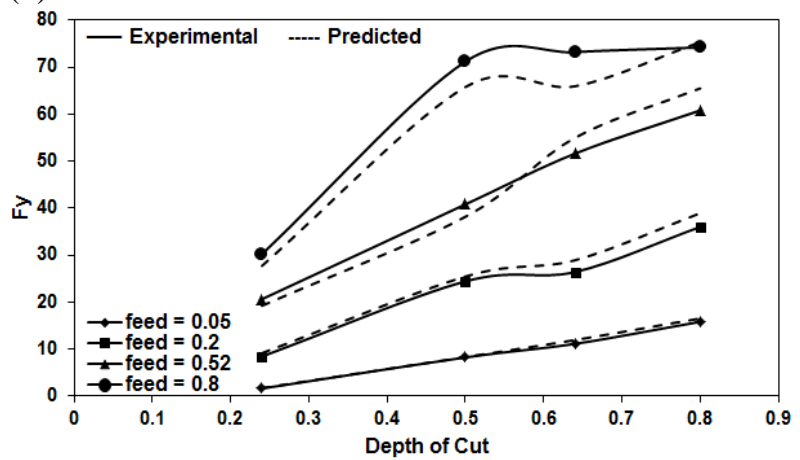

(c)

Fig. 2 Variation of $F_{\mathrm{Y}}$ with (a) Speed and (b) Feed at $\mathrm{d}=0.64 \mathrm{~mm}$ and (c) Depth of Cut at V=550 rpm

neural network architecture was frozen for which (i) functional RMS error was minimum, (ii) minimum number of data sets has a deviation error of $10 \%$, (iii) maximum deviation during testing and training is within $20 \%$, and (iv) minimum variation in RMS functional error during training and testing. Table 2 shows the details of the best fit ANN architecture for cutting force components. Once the network was frozen, it was required to establish the confidence in the network architecture for the prediction of $F_{\mathrm{X}}, F_{\mathrm{Y}}$ or $F_{\mathrm{Z}}$. Therefore, the trained network was used for prediction of $F_{\mathrm{X}}, F_{\mathrm{Y}}$ or $F_{\mathrm{Z}}$ using the 12 validation data sets. 
Table 3 Errors in Prediction of Cutting Force Component

\begin{tabular}{llll}
\hline \multirow{2}{*}{$\begin{array}{l}\text { Corce } \\
\text { Component }\end{array}$} & Mraining & \\
\cline { 2 - 4 } & Max. Error & Max. \% Error & RMSD Error \\
\hline$F_{\mathrm{X}}$ & 3.053 & 23.138 & 1.281 \\
\hline$F_{\mathrm{Y}}$ & 5.240 & 10.322 & 2.915 \\
\hline$F_{\mathrm{Z}}$ & 4.567 & 10.953 & 1.150 \\
\hline & Testing & \\
\hline$F_{\mathrm{X}}$ & 3.287 & 17.611 & 1.451 \\
\hline$F_{\mathrm{Y}}$ & 4.709 & 10.328 & 2.795 \\
\hline$F_{\mathrm{Z}}$ & 0.876 & 7.614 & 1.183 \\
\hline & Validation & & \\
\hline$F_{\mathrm{X}}$ & 2.810 & 17.745 & 1.065 \\
\hline$F_{\mathrm{Y}}$ & 4.333 & 8.597 & 3.160 \\
\hline$F_{\mathrm{Z}}$ & 1.078 & 7.704 & 1.170 \\
\hline
\end{tabular}

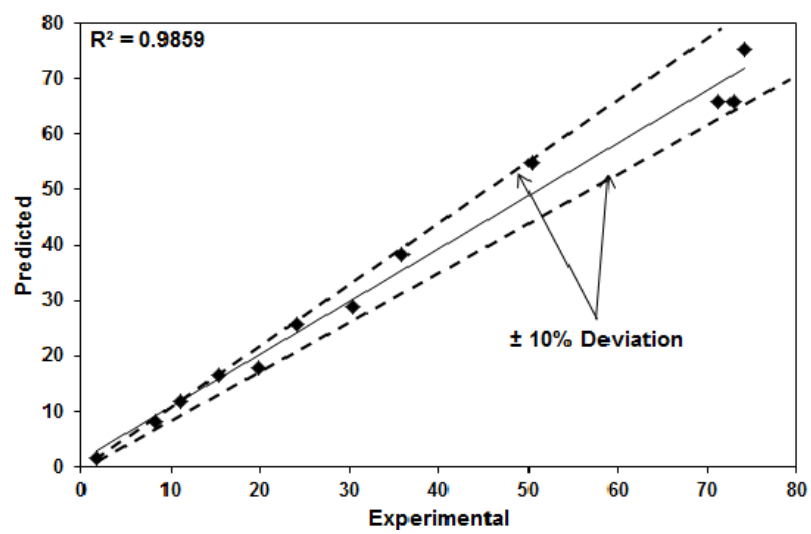

Fig. 3 Variation of predicted cutting force parameters with experimental force parameters for the Validation data set of $F_{\mathrm{Y}}$

Table 3 gives the values of maximum absolute error, maximum percentage error and RMS error calculated for $F_{\mathrm{X}}, F_{\mathrm{Y}}$ and $F_{\mathrm{Z}}$ during training, testing and validation stages separately. Figure 3 shows the plots of experimental vs. predicted values of $F_{\mathrm{Y}}$ during validation. The $F_{\mathrm{X}}$ and $F_{\mathrm{Z}}$ values showed similar prediction accuracy as that of $F_{\mathrm{Y}}$. For perfect prediction, all the points should lie on a line inclined at $45^{\circ}$ to the $x$-axis shown in the figure. Dashed lines representing the boundaries of $\pm 10 \%$ deviations are also shown in the figures. Figures reveal that most of the points lie very close to the line of prefect prediction. The RMS error in the prediction of $F_{\mathrm{Y}}$ is 3.16. The maximum error in the prediction is 4.333 , where the percentage error is $8.597 \%$. The RMS error in the prediction of $F_{\mathrm{X}}$ and $F_{\mathrm{Z}}$ are 1.065 and 1.170 respectively. The maximum errors in the prediction are 2.81 and 1.078 , whereas the percentage errors are $17.745 \%$ and $7.704 \%$ respectively. The above observation shows very good prediction of $F_{\mathrm{X}}, F_{\mathrm{Y}}$ or $F_{\mathrm{Z}}$ by the present ANN modeling. This observation together with the low RMS errors registered, highlight the superior prediction capability of this technique. The prediction also indicates that for most of the cases, the predicted values of the cutting force components hold similar trend with the experimental results. For all the three force components $F_{\mathrm{X}}, F_{\mathrm{Y}}$ and $F_{\mathrm{Z}}$, the predicted cutting force values almost remains constant with increase in cutting speed but increases with increase in feed and depth of cut. Figure 2 shows the close comparison between experimental and predicted values of $F_{\mathrm{Y}}$ for different cutting conditions, revealing the accuracy of the present ANN model within the specified domain. The above ANN modeling may thus be successfully used in formulating the cutting conditions (speed, feed and depth of cut) on the basis of Cutting Forces as demanded by a specific application.

\section{Conclusions}

1) The cutting force components were investigated during orthogonal turning operation for various cutting conditions of speed, feed and depth of cut.

2) The experimental results revealed that the cutting force components almost remain constant with increase in cutting speed while they considerably increase with increase in feed and depth of cut.

3) The cutting force components were modeled as function of three independent cutting parameters viz. speed, feed and depth of cut, by artificial neural network (ANN).

4) The cutting forces subsequently predicted at various cutting conditions within the specified domain have been correlated with the experimental results with fairly good accuracy. During the validation process, it was possible to predict $100 \%, 94 \%$ and $100 \%$ of the cutting force values for $F_{\mathrm{X}}, F_{\mathrm{Y}}$ and $F_{\mathrm{Z}}$, respectively within a percentage deviation of $\pm 10 \%$. This observation highlights the superior prediction capability of ANN technique in the present research area.

\section{References}

X.P. Li, A.Y.C. Nee, Y.S. Wong and H.Q. Zheng, (1999) Theoretical modeling and simulation of milling force, Journal of Material processing Technology, 89, 266. H.Z. Li and X.P. Li, (2002), Milling force prediction using a dynamic shear length model, International, Journal of Machine Tools Manufacturing, 42, 277.

K. Kadirgama, M.M. Noor, N.M. Zuki, M.R.M. Rejab and B. Mohammad, (2008), Prediction Of Cutting Force By Numerical Solution And Statistic Method In End-Milling Operation, KLCC, Kuala lampur, 466.

W.S. Yun and D.W. Cho, (2001), Accurate 3-D cutting force prediction using cutting condition independent coefficients in end milling, International Journal of Machine Tools and Manufacturing, 41, 463.

B. Suksawat, (2010), Chip Form Classification and Main Cutting Force Prediction of Cast Nylon in Turning Operation Using Artificial Neural Network, International Conference on Control, Automation and Systems, Gyeonggi-do, 172.

S. Banerjee, P.S. Robi and A. Srinivasan, (2011), Prediction of High Temperature Flow Behavior of Microalloyed Al-Cu-Mg Alloys by Using Artificial Neural Networks, International Conference on Computational Methods in Manufacturing, IIT Guwahati, 355.

R.K. Jain, (2007), Production Technology, Khanna Publishers, 13, 92.

G.K. Lal, (2007), Introduction to Machining Science, New Age International Publishers, 2, 95.

Y. Altintas, (2000), Manufacturing Automation, Cambridge University Press, 60, 67.

Gokkaya, (2010), The Effects of Machining Parameters on Cutting Forces, Surface Roughness, Built-Up Edge (BUE) and Built-Up Layer (BUL) During Machining AA2014 (T4) Alloy, Journal Of Mechanical Engineering, 56, 589.

P.S. Robi and U.S. Dixit, (2003), Application of neural networks in generating processing map for hot working, Journal of Materials Processing Technology, $142,289$.

Machine Tool Design Handbook, (1982), Tata McGraw-Hill Publishing Company Limited, 12.

K. Satyanarayana, A. Venugopal and G. Venkateshwara Rao, (2011), Effect Of Rake Angle And Feed Rate On Cutting Forces In An Orthogonal Turning Process, ICTMIE, Bangkok.

R.R. Malagi and B.C. Rajesh, (2012), Factors Influencing Cutting Forces in Turning and Development of Software to estimate Cutting Forces in Turning, IJEIT, $2,37$.

L.L.R. Rodrigues, A.N. Kantharaj, B. Kanthraj, W.R.C. Freitas, B.R.N Murthy, (2012), Effect of Cutting Parameters on Surface Roughness and Cutting Force in Turning Mild Steel, Research Journal of Recent Sciences, 1, 19. 